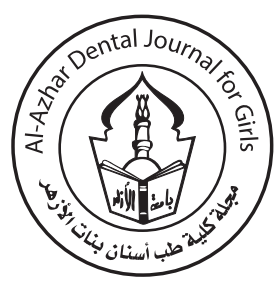

\title{
Role of Expression Ki-67 in Diagnosis of Oral Cancer
}

\author{
Amani A. Mohamed ${ }^{1 *}$, Osama S. EL-Shall ${ }^{2}$ and Naglaa S. EL-Kilani ${ }^{2}$
}

Codex : 55/20.07

azhardentj@azhar.edu.eg

http://adjg.journals.ekb.eg

DOI: $10.21608 /$ adjg.2020.14924.1193

Oral Medicine \& Surgical Sciences (Oral Medicine, Oral \& Maxillofacial Surgery, Oral Pathology, Oral Biology)

\begin{abstract}
Purpose: The study was designed to assess the effect of Ki-67 on oral squamous cell carcinoma (OSCC) with the correlation of biomarker level and grades of OSCC. Material and methods: 26 samples (life biopsies) were inspected in this study, 20 of them are OSCC and 6 are normal. The specimens were processed by the routine histological procedure to prepare them for histopathological and immuno-histochemical examinations. Antibodies expression with anti-Ki-67 antibody was evaluated using image analysis for area percentage with the Leica Q550 image analyzer computer system. Statistical analysis was performed using ANOVA test. Results: Histopathological examination of oral squamous cell carcinoma revealed variable dysplastic changes and alterations of the cells. Ki-67 staining of the poorly differentiated group showed higher immuno-expression than both moderately and well-differentiated groups. The negative expression reveled in control group. The marker positive in cells with OSCC and they were found in the periphery more than the center of the tumor nests, where frequent mitoses were observed. Significant differences were recorded between the control group and all of the experimental groups in Ki-67 expression amounts that represent the area percentage of proliferative cells. Conclusion: $\mathrm{Ki}-67$ is a reliable proliferative marker which can be used for the diagnosis and grading of OSCC transformation.
\end{abstract}

\section{INTRODUCTION}

OSCC is considered the most common malignant tumor that affects the oral cavity. Most of the cases are recorded as OSCC while a small percentage was recorded as, minor salivary gland carcinomas, melano-

Cell Proliferation, Ki-67,

Squamous Cell Carcinoma.

- Paper extracted from Doctor Thesis titled "Role of the Expression of Ki-67 and CD24 Conjugated with Nano- Gold Particles in Diagnosis of Oral Cancer"

1. Assistant lecturer of Oral Medicine , Periodontology, Oral Diagnosis and Dental Radiology department, Faculty of Dentistry, 6 October university, Cairo, Egypt.

2. Professor of Oral Medicine, Periodontology, Oral Diagnosis and Dental Radiology Department, Faculty of Dental Medicine for Girls, Al-Azhar University, Cairo, Egypt.

* Corresponding author email: am_alarabi6@hotmail.com 
mas, sarcomas and metastatic cancers ${ }^{(1)}$. Most of patients diagnosed with oral cancer don't survive longer than 5 years this is due to diagnosis of cancer at later disease stages that affect the treatment outcome ${ }^{(2)}$

Although several treatment modalities are drawn for OSCC including surgery, chemotherapy, radiation or combination of both, its prognosis has not been improved much over the past decades. It is generally believed that prognosis would be more fare if the disease can be detected, diagnosed and treated at its early stages ${ }^{(3)}$.

Molecular markers can be used to predict disease regression and progression. Their use is mandatory to reach a definite diagnosis and treatment of these disorders. Different genes have been shown to be implicated in the progression of oral cancer ${ }^{(4)}$. These include alterations signaling pathway that control cell including apoptosis, angiogenesis and stability of genes. And so, many studies stated that multiple genes and pathways play a role in the mutation from normal to dysplastic stages and finally to oral cancer. That's why it is necessary to assess those alterations in genes ${ }^{(5)}$. Studies reported that many markers are notably correlated with the degree of dysplastic changes ${ }^{(5)}$.

Ki-67 is expressed in $\mathrm{G} 1, \mathrm{~S}, \mathrm{G}_{2}$, and $\mathrm{M}$ phases that were identified as a molecular proliferation marker for the OSCC. Tumor proliferation activity that was reported with the overexpression of $\mathrm{Ki}-67$ in oral cancer is related to a poor prognosis and also is predictive of chemotherapy treatment response ${ }^{(6)}$. The Ki-67 rate is relatively proportion to different histological grading of OSCC and can correlates with clinical and pathological response in oral cancer $^{(7)}$.

The study aimed to evaluate the relationship of $\mathrm{Ki}-67$ expression in oral cancer and to correlate its expression with histological grades of OSCC.

\section{MATERIAL AND METHODS}

\section{Patient Selection}

Twenty six subjects were selected to participate in the present study. Twenty of the patients were selected from the outpatient clinic of National cancer institute, Cairo University, (cancer patient) while the other six patients (control healthy patients) were selected from the Oral Medicine, Periodontology, Oral Diagnosis and Dental Radiology Department, Faculty of Dental Medicine, Girls branch, Al-Azhar University.

After obtaining a satisfactory consent from each subjects denoting their convince about the study protocol, they divided into the following two groups according cancer diagnosis.

Group I: containing 20 patients suffering from oral cancer, (OSCC).

Group II: containing 6 patients having normal tissue as control group.

\section{Tissue sampling:}

Tissue specimens were taken for Group I from the biopsy of OSCC lesions, and for Group II, were excised during gingivectomy and/or orthodontic tooth extraction site. The tissue specimens of both groups were subjected to the following:

- Histopathologic examination using hematoxyclin\& eosin to confirm the provisional diagnosis.

- Immuno-Histopathological using Ki-67.

- Part of specimen was taken for ImmunoBiochemical evaluation using CD24 labeled with gold nanoparticles using Real time PCR

The selected sample won't violate the integrity of the specimen or the final pathology. The total of 20 paraffin-embedded OSCC specimens were classified according to WHO criteria into well, moderate and poorly differentiated. 
Three serial $4 \mu \mathrm{m}$ thickness sections were cut from each sample and formalin-fixed, paraffinembedded tissue block; one section was mounted on ordinary glass slide, for routine stain with $\mathrm{H} \& \mathrm{E}$ to be examined under ordinary light microscope. The other two sections were mounted on positively charged slides for immunostained with anti-Ki-67 antibody to evaluate proliferative activity.

The primary antibody for Ki-67 was monoclonal mouse anti-human Ki-67 oncoprotein (Dako A/S, Glostrup, Denmark; code No M7240) used at a dilution of 1: 100 hat is proved to be the best dilution after the dilution experiments.

Antibody expression was evaluated using Image Analysis for area percentage with positive reaction to Ki-67 was performed using the Leica Q550 image analyzer computer system. All the obtained data from the computer image analysis were then tabulated and statistically evaluated using ANOVA test. The test was considered to give a significant difference if $\mathrm{p}$ value was less than 0.005 .

\section{RESULTS}

\section{Histopathological Findings:}

\section{A. Control Group:}

Microscopic examination of the control specimens revealed normal appearing stratified squamous epithelium and normal appearing underlying connective tissue with no or mild inflammatory cell infiltrate in only 2 of 6 cases.

\section{B. Cases diagnosed as OSCC:}

By light microscopic examination, 9 cases out of 20 OSCC cases were diagnosed as well differentiated, 6 as moderately differentiated and the remaining 5 cases were diagnosed as poorly differentiated tumors.

- Well-differentiated OSCC: All the investigated cases showed sheets and nests of malignant cells resembling squamous epithelium to large extent. The most prominent feature of well differentiated OSCC was the presence of numerous kerain pearls of varying sizes that were more prominent than the epithelial nests. Few dysplastic criteria including nuclear and cellular pleomorphism, nuclear hyperchromatism and altered nuclear cytoplasmic ratio were reported.

- Moderately-differentiated OSCC: All the investigated cases revealed numerous epithelial nests of varying sizes with no kerain pearls. Moderate dysplastic criteria were detected.

- Poorly-differentiated OSCC: All the investigated cases consisted of malignant epithelial cells invading the connective tissue either individually or in small aggregates with great lack of cohesiveness. Those malignant cells bear very little resemblance to their tissue of origin with increased dysplastic criteria. There was no evidence of keratin pearls formation or keratin production.

\section{Immunohistochemical Findings:}

Cases diagnosed as OSCC revealed strong positive Ki-67 immunoexpression compared to control groups which only revealed mild positive nuclear Ki-67 immunoexpression in the basal and suprabasal cells and negative immunoreaction in the most superficial cells.

Immunohistochemical examination of the poorly differentiated group showed a higher expression than both moderately- and well-differentiated groups reflecting more proliferative changes associated with poorly- compared to moderatelyand well-differentiated groups. Ki-67 positivity in OSCC located at the outer surface of the tumor nests than the center of well-differentiated groups.

\section{Statistical Analysis}

Significant difference in mean Ki-67 expression that represents the area $\%$ of proliferative cells was seen between the control group and all of the experimental groups. (Table 1, Fig. 1). The poorlydifferentiated groups showed the highest mean of 
Ki-67 labeling index LI followed by well-differentiated and moderately-differentiated groups.

Table (1) Difference in mean Ki-67 area \% of proliferative cells between control group and experimental groups using ANOVA test

\begin{tabular}{|l|c|c|c|}
\hline \multirow{2}{*}{ Group } & \multicolumn{2}{|c|}{ Area \% } \\
\cline { 2 - 3 } & M \pm Sd & f-Value & p-Value \\
\hline Control & $1.5 \pm 0.5$ & & \\
\cline { 1 - 2 } $\begin{array}{l}\text { Well-differentiated } \\
\text { OSCC }\end{array}$ & $3.6 \pm 1.02$ & & \\
\hline $\begin{array}{l}\text { Moderately- } \\
\text { differentiated OSCC }\end{array}$ & $5.263 \pm 0.8$ & \multirow{2}{*}{$0.0492 *$} \\
\hline $\begin{array}{l}\text { Poorly-differentiated } \\
\text { OSCC }\end{array}$ & $7.6 \pm 0.8$ & & \\
\hline
\end{tabular}

$*$ significant, $(p<0.005)$

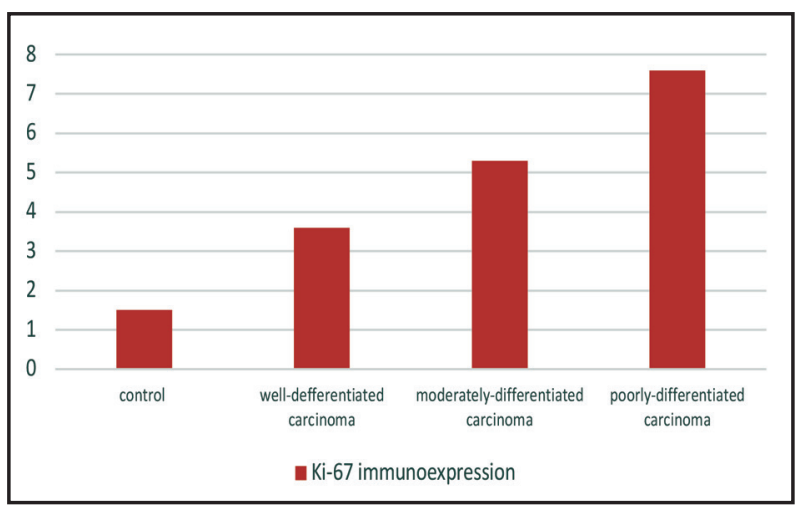

Figure (1): Mean ki67 area \% of proliferative cells between control group and experimental group.

\section{DISCUSSION}

The early cancer detection lead to a fewer side effects from cancer treatments such as radiotherapy and chemotherapy and better prognosis. Furthermore, oral cancer has been reported to have a very high recurrence rate up to a 20 fold ${ }^{(8)}$.

Cell proliferation is most important mechanisms in oncogenesis ${ }^{(9)}$. Various immunohistochemical markers are used to detect cellular proliferation of which $\mathrm{Ki}-67$ is used as a more predictable marker of proliferation in our study.
The monoclonal antibody Ki-67 was first described in 1983 by Johannes Gerdes et al. and was suggested that it might be used as a marker for proliferating cells. Immunostaining with antibodies to $\mathrm{Ki}-67$ antigen is well proven to be a quick and effective method for growth fractions evaluations of various tumor types because of its characteristic reaction patterns that exclusively involves the proliferating cells ${ }^{(10)}$. The Ki-67 antibody was first isolated during an attempt to raise monoclonal antibodies to an antigens specific for Hodgkin and Reed-Sternberg cells ${ }^{(11)}$. Ki-67 are more reliable from other antibodies because it only reacts with cells in their proliferating phase ${ }^{(12)}$.

In the present study, significant difference in mean Ki-67 expression was seen between the control group and all of the experimental groups. The poorly-differentiated groups showed the highest mean of Ki-67 followed by well-differentiated and moderately-differentiated groups. Significant statistically which signify the fact that dysplastic epithelium holds a high potential for malignant transformation and these results were in accordance with other study.

Other study examined the expression of Ki-67 on oral mucosal biopsy samples. They reported that there was upregulation of $\mathrm{Ki}-67$ expression from NOM to dysplasia to OSCC ${ }^{(13)}$. Thus, Ki-67 might be reported as a potential molecular marker in the prognosis of an oral cancer

On contrary, another study reported that no correlation between KI 67 proliferation index in different groups and so expression might be a false prognostic marker of patient with oral cancer. These may be due to sample size and IHC techniques ${ }^{(14)}$.

\section{CONCLUSIONS}

$\mathrm{Ki}-67$ is a reliable proliferative marker that can be used for the diagnosis and grading of OSCC. Information on the growth fraction of the tumors used in detection of tumor grade, and a highly correlation between $\mathrm{Ki}-67$ and the degree of mutation 
has been recorded. Also a marked variation in the Ki-67 expression was observed in different tumors grades, indicating that Ki-67 staining may be used in detection of tumor prognosis.

\section{REFERENCES}

1. Scully C, Felix D. Oral Medicine - Update for the dental practitioner Oral cancer. Br Dent J 2006; 200:13-17.

2. Greenlee RT, Hill-Harmon MB, Murray T. Thun M. Cancer statistics, 2001. CA Cancer J Clin 2001; 51: 15-36.

3. Anastasios K. Current Aspects on Oral Squamous Cell Carcinoma. Open Dent J 2012; 6: 126-30.

4. Nikolaos G, Monica P, Maria G, Catherine F, Douglas E, Paul E, et al. Molecular markers associated with development and progression of potentially premalignant oral epithelial lesions . Oral Surg Oral Med Oral Pathol Oral Radiol 2018;125:650-69.

5. Pitiyage G, Tilakaratne WM, Tavassoli M, Warnakulasuriya S. Molecular markers in oral epithelial dysplasia. J Oral Pathol Med. 2009; 38:737-52.

6. Prihantono P,Mochammad H,Christian B, Daniel S, Haryasena H, Berti N, et al. Ki-67 Expression by Immunohistochemistry and Quantitative Real-Time Polymerase Chain Reaction as Predictor of Clinical Response to Neoadjuvant Chemotherapy in Locally Advanced Breast Cancer. Journal of Oncology 2017; 2017:1- 8 .

7. Amer T,Charif B, Safa Z,Khaled Z, Johnny N, Mahdi A, et al. Ki-67 Prognostic Value in Different Histological Grades of
Oral Epithelial Dysplasia and Oral Squamous Cell Carcinoma Asian Pac J Cancer Prev. 2018; 19:3279-86.

8. Nagler RM. Saliva as a tool for oral cancer diagnosis and prognosis. Oral Oncol. 2009;45: 1006-16.

9. Van Diest PJ, Brugal G, Baak JP. Proliferation markers in tumours: interpretation and clinical value. Journal of clinical pathology. 1998; 51:716-24.

10. Basheer H, Suhas R, Celestina D, Ajay K., Lin J, and Asaf A. Analysis of Ki-67 Expression in Oral Premalignant Lesions and Normal Oral Mucosa: An Immunohistochemical Study. J Pharm Bioallied Sci 2019; 11: 232-35.

11. Smita S, MB R, and Mohsin G, Expression of Ki-67 in normal oral epithelium, leukoplakic oral epithelium and oral squamous cell carcinoma .J Oral Maxillofac Pathol. 2014; 18: 169-76.

12. Iwata H, Masuda N, Sagara Y, Kinoshita T, Nakamura S, Yanagita Y. et al. Analysis of Ki-67 expression with neoadjuvant anastrozole or tamoxifen in patients receiving goserelin for premenopausal breast cancer. Cancer. 2013; 119:704-13.

13. Shang X, Ying L, Xue Q, Rui-X, Kan W, Xiao-F,et al.What is the Prognostic Significance of Ki-67 Positivity in Oral Squamous Cell Carcinoma? Journal of Cancer2016; 7: 758-767.

14. Iwata H, Masuda N, Sagara Y, Kinoshita T, Nakamura S, Yanagita Y.Analysis of Ki-67 expression with neoadjuvant anastrozole or tamoxifen in patients receiving goserelin for premenopausal breast cancer. Cancer. 2013;119: 704-13. 